Интернет-журнал «Отходы и ресурсы» https://resources.today

Russian Journal of Resources, Conservation and Recycling

2020, №1, Tом 7 / 2020, No 1, Vol 7 https://resources.today/issue-1-2020.html

URL статьи: https://resources.today/PDF/18INOR120.pdf

DOI: 10.15862/18INOR120 (http://dx.doi.org/10.15862/18INOR120)

Ссылка для цитирования этой статьи:

Петеляк В.Е., Масленникова О.Е. Мониторинг системы водоснабжения на распределенной производственной территории с помощью беспроводных технологий // Интернет-журнал «Отходы и ресурсы», 2020 №1, https://resources.today/PDF/18INOR120.pdf (доступ свободный). Загл. с экрана. Яз. рус., англ. DOI: 10.15862/18INOR120

For citation:

Petelyak V.E., Maslennikova O.E. (2020). Monitoring the water supply system in a distributed production area using wireless technology. Russian Journal of Resources, Conservation and Recycling, [online] 1(7). Available at: https://resources.today/PDF/18INOR120.pdf (in Russian) DOI: 10.15862/18INOR120

\title{
УДК 004.42
}

\section{ГРНТИ 50.05.19; 50.51.18}

Петеляк Владимир Евстахиевич ФГБОУ ВО «Магнитогорский государственный технический университет им. Г.М. Носова», Магнитогорск, Россия Доцент

Кандидат физико-метаматематических наук, доцент E-mail: petelyak@ya.ru ORCID: http://orcid.org/0000-0001-5202-5224 РИНЦ: https://www.elibrary.ru/author_profile.asp?id=75258

Масленникова Ольга Евгеньевна ФГБОУ ВО «Магнитогорский государственный технический университет им. Г.М. Носова», Магнитогорск, Россия Доцент

Кандидат педагогических наук, доцент E-mail: maslennikovaolga@yandex.ru ORCID: http://orcid.org/0000-0001-6152-2628 РИНЦ: https://www.elibrary.ru/author_profile.asp?id=379300

\section{Мониторинг системы водоснабжения на распределенной производственной территории с помощью беспроводных технологий}

Аннотация. Статья посвящена результатам исследования по выбору способа мониторинга водопроводной сети на распределенной промышленной территории, в частности способам сбора данных с измерительных приборов для обнаружения утечек воды. Мониторинг на большой промышленной территории осложняется множеством факторов, которые затрудняют применение существующих методов обнаружения утечек: сложная топография водопроводной сети, затрудняющая ее сегментирование; изношенность инфраструктуры, как вследствие старения, так и коррозии; разнородность материала изготовления труб; большой разброс диаметров труб в одной трубопроводной системе; разнообразные строительномонтажные и ремонтные работы, ведущиеся на производственной площадке предприятия; значительный уровень круглосуточного производственного шума; наличие неконтролируемых элементов водопроводной сети.

Кроме усложняющих факторов необходимо было учесть заданные ограничения: значительная (более 1 км) удаленность от объектов инфраструктуры; удаленность мест 
контроля от сетей электроснабжения; небольшой объем передаваемых данных; необходимость установки датчиков в водопроводных колодцах, так как невозможно гарантировать сохранность надземных шкафов в производственных условиях.

В качестве возможного решения были рассмотрены беспроводные технологии передачи небольших по объёму данных на большие расстояния, относящих к классу LPWAN (Low-power wide-area network): NB-IoT (Narrow Band Internet of Things), LoRa (Long Range) и «Стриж». Общие преимуществами технологий являются высокая проникающая способность и большой радиус действия, и низкое энергопотребление, позволяющее обеспечить конечным устройствам бесперебойную автономную работу до 10 лет без замены батареи. Также каждая технологии имеет дополнительные преимущества и ограничения.

Вывод исследования является, что для решения задачи в заданных ограничениях можно использовать любую из рассмотренных технологий, при этом при конкретизации задачи необходимо учесть дополнительные параметры, такие как наличие покрытия сети в заданном районе, количество размещаемых датчиков, доступность необходимых устройств, финансовые ограничения.

Ключевые слова: мониторинг; водопроводная система; утечки воды; беспроводные технологии; Low-power wide-area network; Narrow Band Internet of Things; технология Стриж; энергоэффективный протокол; высокая проникающая способность; устойчивая связь на большом расстоянии

\section{Введение}

Промышленные предприятия в своей производственной деятельности потребляют различные ресурсы, в частности, питьевую воду. Питьевая вода может использоваться в технологических процессах, для охраны труда (в санитарно-гигиенических целях) и в системах пожаротушения. Для снабжения водой по территории предприятия прокладываются трубопроводные сети водоснабжения от поставщиков водных ресурсов до конечных потребителей на производственной территории.

При включении предприятия в производственный кластер или городскую инфраструктуру, водный ресурс может транспортироваться через территорию предприятия другим потребителям.

Если производственная территория большая - протяженность трубопроводов может составлять от нескольких десятков до нескольких сотен километров, и значительная часть трубопроводной системы может находиться вне зоны доступа к электрическим сетям и информационной инфраструктуре предприятия.

Как и любой другой ресурс, питьевая вода подлежит контролю и учету. Чаще всего питьевая вода - это внешний ресурс для предприятия, поэтому в первую очередь измерительные приборы устанавливаются в точках поступления воды в производственную зону и точках передачи (точках выхода), если вода транспортируется другим потребителям. Таких точек небольшое количество, измеряемые данные важны для контроля параметров поступающей воды (обычно это расход, давление и температура) и для взаиморасчетов с поставщиками водных ресурсов, поэтому, как правило, эти участки автоматизируются в первую очередь. Также учитывается потребление питьевой воды различными производственными участками и процессами. В зависимости от целей учета, нормативнорегламентирующей документации и уровня автоматизации производства, учет может быть нормативным (по установленным нормам) или приборным (по приборам учета). На некоторых 
производствах, в первую очередь пищевых, для которых критичным является качественный состав питьевой воды, делается регулярный химический анализ воды.

Одной из проблем эксплуатации водопроводов являются течи воды, которые необходимо своевременно обнаруживать и устранять. На больших территориальных производственных площадках, на которых неоднократной реконструкции подвергалась как водопроводная система, так и производственные объекты, задача обнаружения и устранения мест утечек воды требует много времени.

Существуют различные методы обнаружения утечек в водопроводах как техническими средствами [1; 2], так и с помощью математического моделирования [3; 4]. Однако каждый из этих методов имеет ограничения, которые затрудняют или делают невозможными их использование на производственной площадке промышленного предприятия.

Можно выделить следующие факторы, затрудняющие использование существующих методов обнаружения утечек в водопроводах:

- сложная топография водопроводной сети, затрудняющая ее сегментирование;

- изношенность инфраструктуры, как вследствие старения, так и коррозии и образования различных отложений на внутренних стенках труб, что приводит к постоянному изменению параметров трубопроводной системы;

- разнородность материала изготовления труб (чугун, сталь, полимер);

- большой разброс диаметров труб в одной трубопроводной системе;

- $\quad$ трубопроводы с холодной водой расположены ниже глубины промерзания грунта и не могут быть вынесены на поверхность в отличие от теплотрасс;

- $\quad$ разнообразные строительно-монтажные и ремонтные работы, ведущиеся на производственной площадке предприятия;

- $\quad$ значительный уровень производственного шума, часто круглосуточный, что делает невозможным использование акустических методов;

- $\quad$ наличие внутрипроизводственных элементов водопроводной сети, таких как насосы, работа которых по тем или иным причинам не контролируется.

Кроме технических негативных факторов, ограничивающую роль играет экономический фактор - несмотря на то, что питьевая вода - это ценный ресурс, он имеет низкую стоимость. Поэтому способы обнаружения и устранения мест утечек воды должны быть экономически целесообразными, что делает практически невозможным использование в этом случае систем обнаружения утечек, применяемых для обнаружения утечек в нефтегазопроводах и тепловых сетях. Также надо отметить, что утечки в водопроводных системах не несут существенного техногенного риска и опасности для предприятия и не влекут за собой необратимых или дорогостоящих санкций со стороны надзорных органов.

Как отмечается в работе [1], «в настоящее время не существует универсального метода поиска утечки, позволяющего определить любую потерю воды (малую, большую), на трубопроводе из любого материала, поэтому только комплексное использование нескольких методов позволяет точно и быстро определять место утечки». Только наличие данных, полученных разными способами, позволяет сопоставить эти данные, локализовать сначала область утечки, затем, с использованием уже более подходящих для данных условий способов, определить точное место утечки и устранить его. 
Для оперативного получения данных необходимо дополнительно к переносным мобильным измерительным комплексам в ключевых точках выделенных сегментов водопроводной системы иметь стационарные датчики или измерительные приборы и снимать данные с заданной периодичностью. На способы получения данных могут накладываться различные ограничения, вызванные производственными условиями, организационнонормативным регулированием и существующей информационно-коммуникационной инфраструктурой.

\section{Ограничения задачи}

В данной работе принимаются во внимание следующие ограничения:

- $\quad$ значительная (более 1 км) удаленность от объектов инфраструктуры, что влечет необходимость использования беспроводного сбора данных;

- $\quad$ удаленность от сетей электроснабжения, что влечет необходимость обеспечения автономности измерительных приборов;

- н небольшой объем передаваемых данных;

- н необходимость установки датчиков в водопроводных колодцах, так как невозможно гарантировать сохранность надземных шкафов в производственных условиях.

\section{Методы решения}

Технологии беспроводной передачи данных имеют достаточно давнюю историю. Первый открытый международный индустриальный стандарт X10 был разработан еще в 1975 году, а с 90-х годов двадцатого века было разработано множество протоколов и стандартов для различных применений [5]. В рамках данной работы будут рассмотрены только технологии, удовлетворяющие указанным выше ограничениям.

До недавнего времени для решения задач, подобных описанной в данной статье, преимущественно использовались технологии Wi-Fi (на основе стандартов IEEE 802.11), GPRS (General Packet Radio Service, надстройка над технологией мобильной связи GSM, Global System for Mobile Communications), WirelessHART (Wireless Highway Addressable Remote Transducer Protocol, на базе протокола HART). Технология Wi-Fi, при большой пропускной способности, значительно превосходящей потребности задачи, имеет существенный недостаток - ограниченный радиус действия (до 1000 м). Этот недостаток преодолевается установкой повторителей и усилителей сигналов, однако на промышленной площадке сохранность таких устройств, установленных на уровне земли, не может быть обеспечена по производственным причинам. Также недостатком технологии является большое энергопотребление (ведутся работы по снижению энергопотребления технологии). Недостатком технологии WirelessHART также является малый радиус действия. Технология GPRS, в силу повсеместного распространения сотовой связи стандарта GSM на российской территории, позволяет организовать сбор данных с распределенных по территории датчиков. Недостатком технологии GPRS является передача пакетных данных через неиспользуемые в момент передачи голосовые каналы. Российские операторы сотовой связи используют приоритет голосового трафика перед данными, поэтому загрузка сети является критическим фактором для скорости передачи данных и возникновения задержек в передаче данных. Недостатки описанных технологий компенсировались дополнительными программнотехническими решениями. 
Начиная с 2015 года, были разработаны новые беспроводные технологии передачи небольших по объёму данных на большие расстояния, больше подходящие для решения задач в указанных выше ограничениях. Это семейство новых технологий под общим названием LPWAN (Low-power wide-area network, энергоэффективная сеть дальнего радиуса действия) включает:

- $\quad$ NB-IoT (Narrow Band Internet of Things), беспроводная технология на базе инфраструктуры сотовых сетей, разработана консорциумом 3GPP;

- $\quad$ LoRa (Long Range), объединяет в себе проприетарный метод модуляции LoRa на физическом уровне и открытый протокол LoRaWAN (Long Range Wide Area Network), поддерживается некоммерческой организации «LoRa Alliance»;

- «Стриж», на базе проприетарного энергоэффективного радиопротокола связи XNB (Extended Narrowband), разработана российской компанией «Современные Радио Технологии».

Подробное описание этих технологий, сравнительный анализ и примеры их применения можно найти в [6-12]. Каждая технология имеет свои преимущества и ограничения.

\section{Результаты}

Технология NB-IоT развивается сотовыми операторами ${ }^{1,2,3}$ в лицензируемом диапазоне частот, выделенном Государственной комиссией по радиочастотам (ГКРЧ) в декабре 2017 года 4 . Особенности технологии:

- $\quad$ использование существующей инфраструктуры, пока зона покрытия охватывает, в основном крупные города (текущие зоны покрытия указаны на сайтах сотовых операторов), но в перспективе, сравнится с зоной покрытия GSM;

- привязка к операторским услугам;

- $\quad$ низкое энергопотребление позволяет автономным датчикам NB-IoT работать до 10 лет без замены батареи (зависит от режима использования);

- $\quad$ каждое автономное устройство должно имеет SIM-карту (Subscriber Identification Module) c ежемесячной или ежегодной тарифной платой, что в решениях c большим количеством устройств может привести к большим постоянным затратам.

Развитием технологии LoRa в России занимаются такие компании, как «ER-Telecom» (www.iot-ertelecom.ru), «MTT» (www.mtt.ru), «Лартех» (lar.tech), некоммерческая «Ассоциация Интернета вещей» (комитет LoRaWAN, iotas.ru/committee/lora-lpwan), другие участники рынка. Особенности технологии:

${ }^{1}$ Интернет вещей IоT/M2М. Режим доступа: https://moskva.mts.ru/business/internet-veshheyiotm2m/upravlenie-m2m-ustroystvami (дата обращения: 01.03.2020).

${ }^{2}$ Отраслевые решения IоT. Режим доступа: https://moskva.beeline.ru/business/products-andsolutions/monitoring/otraslevye-resheniia-iot (дата обращения: 01.03.2020).

${ }^{3}$ IoT/M2M-решения для бизнеса. Режим доступа: https://iot.megafon.ru (дата обращения: 01.03.2020).

4 Заседание ГКРЧ от 28 декабря 2017 года (протокол №17-44). Режим доступа: https://digital.gov.ru/ru/documents/5875 (дата обращения: 01.03.2020). 
- $\quad$ открытость протокола LoRaWAN, что позволяет множеству разработчиков предложить большой спектр разнообразных устройств по низкой цене;

- $\quad$ компоненты технологии от разных производителей не являются полностью заменяемыми;

- $\quad$ использование нелицензированного диапазона частот (в России диапазон частот технологии LoRaWAN ограничен по сравнению с международным диапазоном, что не позволяет в полной мере использовать оборудование, производимое международными компаниями);

- $\quad$ высокая проникающая способность радиосигнала в зданиях и закрытых помещениях, устойчивая связь на расстоянии до 5 км в городских условиях при плотной застройке и свыше 15 км в сельской местности (при прямой видимости);

- $\quad$ низкое собственное энергопотребление конечных устройств, заявленное время автономной работы конечных может достигать 10 лет;

- можно создавать собственные сети или использовать сети поставщиков;

- высокая масштабируемость сети на больших территориях;

• задержка передачи данных может достигать нескольких секунд;

- $\quad$ высокая вероятность коллизий при большом количестве устройств в районе одной базовой станции.

Технология «Стриж» разработана и поддерживается отечественной компанией «Современные Радио Технологии» (strij.tech). Особенности технологии:

- $\quad$ полностью проприетарная (закрытая) технология;

• $\quad$ использование нелицензированного диапазона частот;

- $\quad$ собственный узкополосный энергоэффективный радиопротокол позволяет эффективнее использовать спектр частот и снизить вероятность коллизий;

- высокая проникающая способность и радиус действия до 10 км;

- $\quad$ заявленная автономность работы датчиков составляет до 10 лет без замены батареи;

- $\quad$ компания предлагает весь спектр технологического оборудования (радиомодули, базовые станции, конечные устройства), что позволяет обеспечить полную совместимость разного оборудования, но существенно ограничивает его разнообразие;

- общая низкая стоимость решения;

- она покрытия технологий «Стриж» на коней 2019 года составляет 45 российских регионов.

\section{Заключение}

Для решения задач в заданных ограничениях принципиально подойдет любая из рассмотренных технологий, при этом надо учитывать такие дополнительные параметры, как наличие покрытия сети в заданном районе, количество размещаемых датчиков, доступность необходимых устройств, финансовые ограничения. 


\section{ЛИТЕРАТУРА}

1. Косыгин А.Б., Ханин В.Н., Государев К.И., Фомина И.В. Обнаружение скрытых утечек с использованием системы мониторинга водопроводной сети // Водоснабжение и санитарная техника. 2010. № 4. С. 22-26.

2. Орлов Е.В., Комаров А.С., Мельников Ф.А., Серов А.Е. Утечки в трубопроводах систем внутреннего водоснабжения // Вестник МГСУ. 2015. № 3. С. 40-47.

3. Аширова О.А., Салохиддинов А.Т. Метод эффективного поиска скрытых мест утечек воды в подземных трубопроводах // Научный журнал Российского НИИ проблем мелиорации. 2017. № 2 (26). С. 156-170.

4. Егорова Ю.А., Коневский Е.В., Васьковский А.В., Зайко В.А. Опыт использования геоинформационных технологий и программно-расчетных комплексов при поиске неучтенных потерь воды в системе водоснабжения г. Самары // Водоснабжение и санитарная техника. 2016. № 8. С. 45-50.

5. Колыбельников А.И. Обзор технологий беспроводных сетей // Труды МФТИ. 2012. T. 4. № 2 (14). C. 3-29.

6. Рентюк В. Краткий путеводитель по беспроводным технологиям «Интернета вещей». Часть 4. Большой радиус действия // Control Engineering Россия. 2018. №3 (75). C. 82-87.

7. Верхулевский К. Особенности и тенденции развития технологии LoRaWAN // Беспроводные технологии. 2017. № 1. С. 12-18.

8. Алексеев В. Технологии «Интернета вещей» для сетей ISM нелицензируемого диапазона частот // Беспроводные технологии. 2017. № 1. С. 44-50.

9. Смирнов A. LoRaWAN над Санкт-Петербургом // Беспроводные технологии. 2017. № 4. C. 58-61.

10. Роенков Д.Н., Яронова Н.В. Основы технологии LoRa. Перспективы еe применения // Автоматика, Связь, Информатика. 2017. № 4. С. 31-35.

11. Скородумов А. Учет ресурсов по сетям LoRaWAN: решения от компании «ЕвроМобайл» // Беспроводные технологии. 2018. № 4. С. 48-51.

12. Tokmakov D., Sotirov S., Gluhov S. Lorawan based system for measurment and monitoring of temperature and humidity in data centers and server rooms // Научни трудове на съюза на учените - Пловдив. Серия В: Техника и технологии - 2019. T 17. C. 165-168. 
Petelyak Vladimir Evstakhievich

Nosov Magnitogorsk state technical university, Magnitogorsk, Russia

E-mail: petelyak@ya.ru

Maslennikova Olga Evgen'evna

Nosov Magnitogorsk state technical university, Magnitogorsk, Russia E-mail: maslennikovaolga@yandex.ru

\title{
Monitoring the water supply system in a distributed production area using wireless technology
}

\begin{abstract}
The article is devoted to the results of a study on the choice of a method for monitoring the water supply network in a distributed industrial area, in particular, methods for collecting data from measuring instruments for detecting water leaks. Monitoring over a large industrial area is complicated by many factors that impede the use of existing leak detection methods: complex topography of the water supply network, which makes it difficult to segment; deterioration of infrastructure, both due to aging and corrosion; heterogeneity of the material of the manufacture of pipes; large variation in pipe diameters in one pipeline system; a variety of construction, installation and repair work carried out at the production site of the enterprise; significant level of round-the-clock production noise; the presence of uncontrolled elements of the water supply network.

In addition to complicating factors, it was necessary to take into account the specified limitations: a significant distance from the infrastructure; remoteness of control places from power supply networks; small amount of transmitted data; the need to install sensors in water wells, since it is impossible to guarantee the safety of elevated cabinets in a production environment.
\end{abstract}

As a possible solution, we considered wireless technologies for transmitting small-volume data over long distances belonging to the class of LPWAN (Low-power wide-area network): NB-IoT (Narrow Band Internet of Things), LoRa (Long Range) and Strizh. The common advantages of the technology are high penetration and long range and low power consumption, which allows end devices to provide uninterrupted autonomous operation for up to 10 years without replacing the battery. Each technology also has additional advantages and limitations.

The conclusion of the study is that to solve the problem in the given restrictions, you can use any of the technologies considered, while specifying the problem, you need to take into account additional parameters, such as the availability of network coverage in a given area, the number of sensors to be placed, the availability of the necessary devices, financial constraints.

Keywords: monitoring; plumbing system; water leakage; wireless technology; Low-power wide-area network; Narrow Band Internet of Things; Swift technology; energy-efficient protocol; high penetration; stable long-distance communications 


\section{REFERENCES}

1. Kosygin A.B., Khanin V.N., Gosudarev K.I., Fomina I.V. (2010). Detection of hidden leaks using a monitoring system of the water supply network. Water supply and sanitary equipment, 4, pp. 22-26 (in Russian).

2. Orlov E.V., Komarov A.S., Mel'nikov F.A., Serov A.E. (2015). Leaks in pipelines of domestic water supply systems. Bulletin of Moscow State University of Civil Engineering, 3, pp. 40-47 (in Russian).

3. Ashirova O.A., Salokhiddinov A.T. (2017). Method for the effective search for hidden places of water leaks in underground pipelines. Scientific journal of the Russian Research Institute of Land Reclamation, 2(26), pp. 156-170 (in Russian).

4. Egorova Yu.A., Konevskiy E.V., Vas'kovskiy A.V., Zayko V.A. (2016). The experience of using geoinformation technologies and software and calculation systems when searching for unaccounted water losses in the water supply system of Samara. Water supply and sanitary equipment, 8, pp. 45-50 (in Russian).

5. Kolybel'nikov A.I. (2012). Overview of wireless networking technologies. Transactions of Moscow Institute of Physics and Technology, 2(4), pp. 3-29 (in Russian).

6. Rentyuk V. (2018). A short guide to the wireless technology of the Internet of Things. Part 4. Long range. Control Engineering Russia, 3(75), pp. 82-87 (in Russian).

7. Verkhulevskiy K. (2017). Features and development trends of LoRaWAN technology. Wireless Technologies, 1, pp. 12-18 (in Russian).

8. Alekseev V. (2017). "Internet of Things" technologies for ISM networks of unlicensed frequency range. Wireless Technologies, 1, pp. 44-50 (in Russian).

9. Smirnov A. (2017). LoRaWAN over St. Petersburg. Wireless Technologies, 4, pp. 5861 (in Russian).

10. Roenkov D.N., Yaronova N.V. (2017). Basics of LoRa technology. Prospects for its use. Automation, Communication, Informatics, 4, pp. 31-35 (in Russian).

11. Skorodumov A. (2018). Resource accounting for LoRaWAN networks: solutions from EuroMobile. Wireless Technologies, 4, p. 48-51 (in Russian).

12. Tokmakov D., Sotirov S., Gluhov S. (2019). Lorawan based system for measurment and monitoring of temperature and humidity in data centers and server rooms. Scientific Papers of the Union of Scientists - Plovdiv. Series B: Engineering and Technology, (17), pp. 165-168. 